\title{
Formulation of a Novel Nanoemulsion System for Enhanced Solubility of a Sparingly Water Soluble Antibiotic, Clarithromycin
}

\author{
Stuti Vatsraj, ${ }^{1}$ Kishor Chauhan, ${ }^{2}$ and Hilor Pathak ${ }^{1}$ \\ ${ }^{1}$ P. D. Patel Institute of Applied Sciences, Charotar University of Science \& Technology (CHARUSAT), Changa, Gujarat 388 421, India \\ ${ }^{2}$ Department of Integrated Biotechnology, Ashok \& Rita Patel Institute of Integrated Studies and Research in Biotechnology and Allied \\ Sciences (ARIBAS), Sardar Patel University, Vallabh Vidyanagar, Gujarat 388120, India
}

Correspondence should be addressed to Hilor Pathak; hilorpathak.mi@charusat.ac.in

Received 21 August 2013; Revised 7 January 2014; Accepted 7 January 2014; Published 23 March 2014

Academic Editor: Sanjeeb K. Sahoo

Copyright @ 2014 Stuti Vatsraj et al. This is an open access article distributed under the Creative Commons Attribution License, which permits unrestricted use, distribution, and reproduction in any medium, provided the original work is properly cited.

\begin{abstract}
The sparingly water soluble property of majority of medicinally significant drugs acts as a potential barrier towards its utilization for therapeutic purpose. The present study was thus aimed at development of a novel oil-in-water $(\mathrm{o} / \mathrm{w})$ nanoemulsion (NE) system having ability to function as carrier for poorly soluble drugs with clarithromycin as a model antibiotic. The therapeutically effective concentration of clarithromycin, $5 \mathrm{mg} / \mathrm{mL}$, was achieved using polysorbate 80 combined with olive oil as lipophilic counterion. A three-level three-factorial central composite experimental design was utilized to conduct the experiments. The effects of selected variables, polysorbate 80 and olive oil content and concentration of polyvinyl alcohol, were investigated. The particle size of clarithromycin for the optimized formulation was observed to be $30 \mathrm{~nm}$. The morphology of the nanoemulsion was explored using transmission electron microscopy (TEM). The emulsions prepared with the optimized formula demonstrated good physical stability during storage at room temperature. Antibacterial activity was conducted with the optimized nanoemulsion NESH 01 and compared with free clarithromycin. Zone of inhibition was larger for NESH 01 as compared to that with free clarithromycin. This implies that the solubility and hence the bioavailability of clarithromycin has increased in the formulated nanoemulsion system.
\end{abstract}

\section{Introduction}

The increasing frequency of poorly soluble new chemical entities exhibiting therapeutic activity is of major concern to the pharmaceutical industry [1]. These drugs are difficult to process or administer to patients due to poor dissolution. This is the major hurdle that prevents the commercialization of poorly water soluble drugs. There are various techniques available to improve the solubility of such drugs. One of them includes formulation of emulsions using a biocompatible emulsifier to disperse oil in an aqueous phase and is widely used for drug delivery [2-6]. The emulsions are usually biocompatible, biodegradable, physically stable (particularly nanoemulsions and microemulsions), and relatively easy to produce on a large scale [7].

Nanoemulsions (NEs) can be defined as nonequilibrium, heterogeneous systems consisting of two immiscible liquids in which one liquid is dispersed in another liquid as droplets with diameters of tens to a few hundred nanometers [8]. The major advantages of nanoemulsions as drug delivery carriers include ease of fabrication, increased drug loading, enhanced drug solubility and bioavailability, reduced patient variability, controlled drug release, and protection from enzymatic degradation [8].

Clarithromycin (CLA) is a semisynthetic macrolide antibiotic having a 14-membered ring (Figure 1). The chemical structure of CLA differs from erythromycin at position 6 on the lactone ring where the hydroxyl group of later is replaced by methyl group. These chemical modifications rendered CLA acid stable having an increased spectrum of activity, better pharmacokinetic properties, and fewer gastrointestinal adverse effects than erythromycin [9]. Since it is acid stable, clarithromycin is also used to treat the infections in the gastrointestinal tract. However, CLA depicts poor 


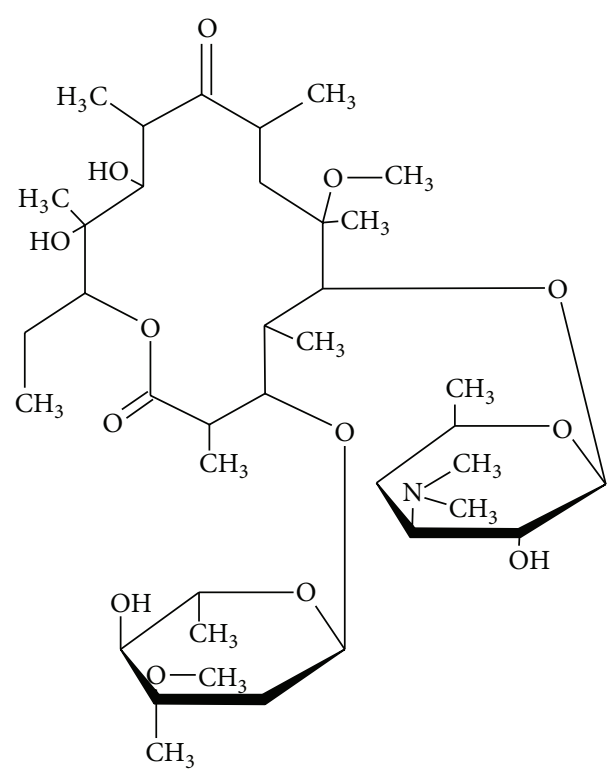

FIGURE 1: Structure of clarithromycin.

water solubility $(0.34 \mathrm{mg} / \mathrm{L})$ which is the major hindrance for its therapeutic usage.

Very sparse studies have been reported towards enhancement of the solubility of CLA. A tocol emulsion for the intravenous delivery of CLA was prepared and demonstrated a marked pain reduction [7]. However, the concentration of drug in the formulation was $2.5 \mathrm{mg} / \mathrm{mL}$ which was not high enough to be therapeutically useful. A tocol emulsion using tocopherol succinate (TS) as lipophilic counterions to improve CLA solubility was reported by Constantinides et al. [10]. However, relatively high content of tocopherol and the need for bioincompatible surfactants for formulation led to regulatory issues [11].

Taking into consideration the above concerns, the present study was aimed at increasing the solubility of sparingly water soluble clarithromycin drug by fabrication of oil in water nanoemulsion system and investigating its antibacterial activity.

\section{Materials and Methods}

2.1. Chemicals. Clarithromycin (CLA) as active pharmaceutical ingredient (API) was a kind gift from Astron Research Limited, Ahmedabad, India. Polysorbate 80 and polyvinyl alcohol were procured from Himedia (Mumbai, India). Purified olive oil and squalene were procured from Merck (Germany). All reagents used in the present study were of analytical grade.

2.2. Formulation of Oil in Water $(\mathrm{O} / \mathrm{W})$ Nanoemulsion. For the formulation of oil in water $(\mathrm{O} / \mathrm{W})$ nanoemulsion, oil phase was constructed using olive oil, squalene, Triton $\mathrm{X}$ 100 , and polysorbate 80 as shown in Table 1 . The oil phase was further mixed using a cyclomixer for 20 minutes. The aqueous phase consisted of (0.1\%) polyvinyl alcohol or
TABLE 1: Range of values for the response surface method.

\begin{tabular}{lccccc}
\hline Independent variables & \multicolumn{5}{c}{ Levels } \\
& $-\alpha$ & -1 & 0 & +1 & $+\alpha$ \\
\hline Olive oil volume $(\mu \mathrm{L})$ & -163.7 & 5 & 252.5 & 500 & 668.7 \\
Polysorbate $80(\mu \mathrm{L})$ & 159.1 & 500 & 1000 & 1500 & 1840.8 \\
Polyvinyl alcohol $(\%)$ & -0.20 & 0.1 & 0.55 & 1 & 1.3 \\
\hline
\end{tabular}

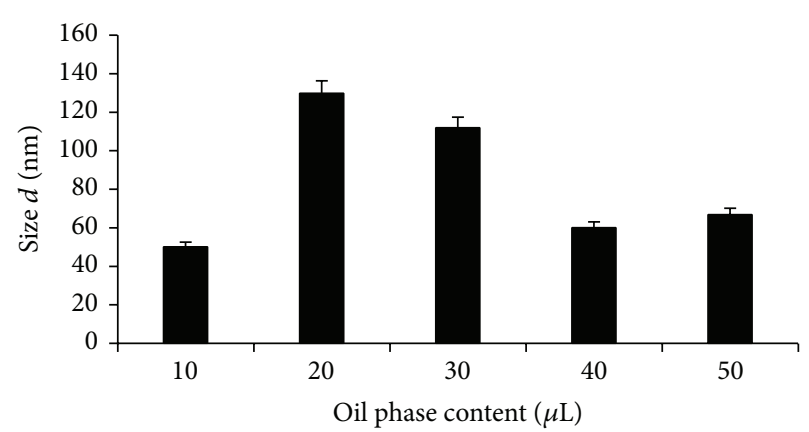

FIGURE 2: Optimization of oil phase content for nanoemulsion system.

(0.1\%) polyethylene glycol. $10 \mu \mathrm{L}$ of the above oil phase was subsequently added to $550 \mu \mathrm{L}$ of aqueous phase and sonicated using an ultrasonicator bath for 10 minutes.

2.3. Fabrication of NESH 01 Containing CLA. NESH 01 (Table 1) was further used in the present study. NESH 01 nanoemulsion is an oil-in-water emulsion manufactured from ingredients that are on the FDA list of recognized ingredients in drug substances. The emulsion was fabricated using olive oil, polysorbate 80 , clarithromycin, polyvinyl alcohol, and water. For fabrication of the nanoemulsion, the lipid phase comprised CLA dissolved in olive oil $(5 \mathrm{mg} / \mathrm{mL})$ and polysorbate $80.10 \mu \mathrm{L}$ of this phase was added to aqueous phase which comprised $550 \mu \mathrm{L}$ of $0.1 \%$ polyvinyl alcohol. All the ingredients were subjected to vortexing followed by ultrasonication (Sonics VC505, Vibra Cell, USA) for $10 \mathrm{~min}$. Optimization of oil phase content in the nanoemulsion was performed as shown in Figure 2. The nanoemulsion obtained in this manner was subjected to further characterization.

\subsection{Characterization of NESH 01}

2.4.1. Transmission Electron Microscopy (TEM). Morphology and structure of the nanoemulsions were studied using transmission electron microscopy (Jeol, JEM 2100, Tokyo, Japan) operating at $200 \mathrm{KV}$ and capable of point-to-point resolution. Combination of bright field imaging at increasing magnification and of different diffraction modes was used to reveal the form and size of nanoemulsion droplets. In order to perform the TEM observations, a drop of the nanoemulsion was directly deposited on the copper coated film grid and observed after drying.

2.4.2. Nanoemulsion Droplet Size Analysis. Droplet size distribution of the nanoemulsion was determined by photon 
TABLE 2: Experimental setup for 20 experiments.

\begin{tabular}{|c|c|c|c|c|c|c|}
\hline \multirow{2}{*}{ Std. run } & \multicolumn{2}{|c|}{ Olive oil $(\mu \mathrm{L})$} & \multicolumn{2}{|c|}{ Polysorbate $80(\mu \mathrm{L})$} & \multicolumn{2}{|c|}{ PVA (\%) } \\
\hline & Coded & Actual & Coded & Actual & Coded & Actual \\
\hline 1 & -1.000 & 5 & -1.000 & 500 & -1.000 & 0.1 \\
\hline 2 & 1.000 & 500 & -1.000 & 500 & -1.000 & 0.1 \\
\hline 3 & -1.000 & 5 & 1.000 & 1500 & -1.000 & 0.1 \\
\hline 4 & 1.000 & 500 & 1.000 & 1500 & -1.000 & 0.1 \\
\hline 5 & -1.000 & 5 & -1.000 & 500 & 1.000 & 1 \\
\hline 6 & 1.000 & 500 & -1.000 & 500 & 1.000 & 1 \\
\hline 7 & -1.000 & 5 & 1.000 & 1500 & 1.000 & 1 \\
\hline 8 & 1.000 & 500 & 1.000 & 1500 & 1.000 & 1 \\
\hline 9 & -1.682 & -163.744 & 0.000 & 1000 & 0.000 & 0.55 \\
\hline 10 & 1.682 & 668.7437 & 0.000 & 1000 & 0.000 & 0.55 \\
\hline 11 & 0.000 & 252.5 & -1.682 & 159.1036 & 0.000 & 0.55 \\
\hline 12 & 0.000 & 252.5 & 1.682 & 1840.896 & 0.000 & 0.55 \\
\hline 13 & 0.000 & 252.5 & 0.000 & 1000 & -1.682 & -0.20681 \\
\hline 14 & 0.000 & 252.5 & 0.000 & 1000 & 1.682 & 1.306807 \\
\hline 15 & 0.000 & 252.5 & 0.000 & 1000 & 0.000 & 0.55 \\
\hline 16 & 0.000 & 252.5 & 0.000 & 1000 & 0.000 & 0.55 \\
\hline 17 & 0.000 & 252.5 & 0.000 & 1000 & 0.000 & 0.55 \\
\hline 18 & 0.000 & 252.5 & 0.000 & 1000 & 0.000 & 0.55 \\
\hline 19 & 0.000 & 252.5 & 0.000 & 1000 & 0.000 & 0.55 \\
\hline 20 & 0.000 & 252.5 & 0.000 & 1000 & 0.000 & 0.55 \\
\hline
\end{tabular}

correlation spectroscopy, which analyzes the fluctuations in light scattering due to Brownian motion of the particles, using a Zetasizer S-90 1000 HS (Malvern Instruments, UK). Light scattering was monitored at $25^{\circ} \mathrm{C}$ at a $90^{\circ}$ angle. Droplet size distribution studies were performed at a fixed refractive index of the respective formulation. The $\mathrm{O} / \mathrm{W}$ emulsions were diluted 1:200 with water before the measurement.

2.4.3. Refractive Index. Refractive index of nanoemulsion formulation NESH 01 was determined using an Abbes-type refractometer.

2.5. Optimization of Nanoemulsion Formulation by Response Surface Methodology (RSM). Response surface methodology (RSM) was used to evaluate the individual as well as the combined effect of ratio of nanoemulsion ingredients like olive oil, polysorbate 80 , and polyvinyl alcohol concentration using the central composite design (CCD). According to this design the total number of experimental combinations was $2^{k}+2 k+n_{0}$, where $k$ is the number of independent variables and $n_{0}$ is number of repetition of experiments at the center point. The "Design-Expert" version 8.0.4.1, StateEase Inc., Minneapolis, USA, was used for experimental design, regression, and graphical analysis of the data obtained [12-14]. Volumes of olive oil, polysorbate 80 , and polyvinyl alcohol concentration were studied at five different levels (Table 1).

A set of 20 experiments was performed. The minimum and maximum ranges of variables were used and the full experiment design with respect to their values was generated
(Table 2). The data obtained from RSM was subjected to the analysis of variance (ANOVA) and the results of RSM were used to fit a second-order polynomial equation:

$$
\begin{aligned}
Y= & \beta_{0}+\beta_{1} A+\beta_{2} B+\beta_{3} C+\beta_{1} \beta_{2} A B+\beta_{1} \beta_{3} A C \\
& +\beta_{2} \beta_{3} B C+\beta_{1} \beta_{1} A^{2}+\beta_{2} \beta_{2} B^{2}+\beta_{3} \beta_{3} C^{2},
\end{aligned}
$$

where $Y$ is response variable (dependent variable), $\beta_{0}$ is intercept (constant), $\beta_{1}, \beta_{2}$, and $\beta_{3}$ are linear coefficients, $\beta_{1} \beta_{2}, \beta_{1} \beta_{3}$, and $\beta_{2} \beta_{3}$ are interaction coefficients, $\beta_{1} \beta_{1}, \beta_{2} \beta_{2}$, and $\beta_{3} \beta_{3}$ are squared coefficients, and $A, B, C, A B, A C, B C$, $A^{2}, B^{2}$, and $C^{2}$ are levels of independent variables. Statistical significance of the above model equation was determined by Fisher's test value, and the proportion of variance explained by the model was given by the multiple coefficient of determination, $R$ squared $\left(R^{2}\right)$ value. The response variable $Y$, in the present study, is the droplet size diameter and its desirable value is $<100 \mathrm{~nm}$ for formulation of oil-in-water nanoemulsion.

\subsection{Stability Studies}

2.6.1. Temporal Stability. The temporal study was done for a period of 7 days by measurement of the droplet diameter size and refractive index of the nanoemulsions at an interval of 24 hours. The nanoemulsion was stored at room temperature until further usage.

2.6.2. Temperature Stability. To investigate the thermal stability of the nanoemulsion, the system NESH 01 was incubated 
for one hour at the temperature range from $20^{\circ} \mathrm{C}$ to $100^{\circ} \mathrm{C}$ followed by the measurement of the droplet diameter of the nanoemulsions.

2.7. Antibacterial Activity of Fabricated Nanoemulsion. The bacterial isolates Bacillus subtilis MTCC 121, Bacillus megaterium MTCC 428, Enterobacter aerogenes MTCC 111, Streptococcus pneumoniae MTCC 655, Azotobacter chrococcus MTCC 446, and Escherichia coli MTCC 1610 were procured from MTCC Chandigarh, India, for the experimental purpose.

Antibacterial activity of the fabricated nanoemulsion NESH-01 was determined using standard spread plate method [13]. Hundred microliters of nanoemulsion was added to the wells on these plates, incubated at $4^{\circ} \mathrm{C}$ for 30 minutes, and then incubated at $37^{\circ} \mathrm{C}$. Plates were observed for zone of growth inhibition around the wells after $24 \mathrm{~h}$ of incubation. Free clarithromycin $(0.65 \mathrm{mg})$ was used as positive control. The nanoemulsion without antibiotic was used as negative control.

\section{Results and Discussion}

3.1. Formulation of the Nanoemulsion Systems. The average diameter of the nanoemulsion systems is as depicted in Table 3. The droplet size of $\mathrm{O} / \mathrm{W}$ emulsions ranged from 68 to $380 \mathrm{~nm}$. The size analysis showed a mean diameter of $68 \mathrm{~nm}$ for olive oil-loaded emulsions (NESH 01). Replacement of the olive oil by squalene (NESH 06) led to a drastic increase in the size from 68 to $293 \mathrm{~nm}$. Addition of sodium dodecyl sulfate to NESH 01 system gave NESH 03 with a mean droplet diameter of $100 \mathrm{~nm}$. The replacement of polysorbate 80 with Triton X 100 to olive oil emulsions produced system $\mathrm{NESH} 02$ with a size of $125 \mathrm{~nm}$. Replacement of polyvinyl alcohol with polyethylene glycol produced system NESH 04 with a mean droplet diameter of $221 \mathrm{~nm}$ and $380 \mathrm{~nm}$ for the system NESH 05 which contained squalene in place of olive oil. It is well established that droplet size imparts significant influence on the bioavailability of nanoemulsions [8]. Therefore, from the above studies, the nanoemulsion system NESH 01 which produced smallest average droplet diameter was further characterized and used in the present study.

3.2. Fabrication of NESH 01. The nanoemulsion was fabricated from FDA approved ingredients, namely, olive oil, polysorbate 80 , clarithromycin, polyvinyl alcohol, and water. CLA at a concentration of $5 \mathrm{mg} / \mathrm{mL}$ was dissolved in olive oil. Olive oil provides better alternative for enhancing the solubility of the antibiotics when compared to pure long chain triglycerides as these pure compounds are more expensive than olive oil. Moreover, olive oil mainly comprises long chain triglycerides-palmitic acid and oleic acid which portray rational solubility for CLA. Li et al. [7] reported the tocopherol succinate nanoemulsion and enhanced the solubility of CLA up to $5 \mathrm{mg} / \mathrm{mL}$. A comparable solubility was achieved with the use of olive oil in the present study.
TABLE 3: Formulation of O/W nanoemulsion systems.

\begin{tabular}{lcc}
\hline Code & O/W nanoemulsion system & $\begin{array}{c}\text { Droplet } \\
\text { diameter }(\mathrm{nm})\end{array}$ \\
\hline NESH 01 & PVA + Polysorbate 80 + Olive oil & $68 \pm 3$ \\
NESH 02 & PVA + Triton X 100 + Olive oil & $125 \pm 5$ \\
NESH 03 & PVA + SDS + Polysorbate 80 + Olive oil & $100 \pm 6$ \\
NESH 04 & PEG + Polysorbate 80 + Olive oil & $220 \pm 2$ \\
NESH 05 & PEG + Polysorbate 80 + Squalene & $380 \pm 7$ \\
NESH 06 & PVA + Polysorbate 80 + Squalene & $293 \pm 3$ \\
\hline
\end{tabular}

PVA: polyvinyl alcohol; PEG: polyethylene glycol; SDS: sodium dodecyl sulfate.

The ability of surfactant molecules to give the necessary curvature of the interfacial surface required to form fine micelles has been associated with the packaging geometry. This refers to the ratio between hydrocarbon volumes, optimum head group area, and tail length of the molecule at the interface [15]. In the present study polysorbate 80 is used as an emulsifier. It comprises oleic acid and polyethoxylated sorbitan. The oleic acid acts as surplus lipophilic counterion that ameliorates the physicochemical properties of the oil phase, resulting in an enhanced solubilizing capacity for CLA. Moreover, to the best of our knowledge, this is the first report on formulation of a nanoemulsion system using olive oil, polysorbate 80 , and polyvinyl alcohol.

3.3. Influence of the Oil Phase Content. Clarithromycin being a poorly water soluble antibiotic; this antibiotic was dissolved in olive oil. Different concentrations of clarithromycin (1$10 \mathrm{mg}$ ) were added to $1500 \mu \mathrm{L}$ of oil phase to determine its solubility and $5 \mathrm{mg}$ of the drug was found to be completely soluble in oil phase (data not shown). To optimize the oil phase content, different volumes of oil phase $(10,20,30,40$, and $50 \mu \mathrm{L})$ were added to aqueous phase $(500 \mu \mathrm{L})$. Figure 2 depicts that increase in the volume of oil phase led to an increase in the mean droplet diameter of the nanoemulsion. This observation can be accredited to the increase in the interfacial surface caused by increasing the oil volume. A similar observation was reported by $\mathrm{He}$ et al. [8] with protein stabilized nanoemulsions. A volume of $10 \mu \mathrm{L}$ oil phase in $550 \mu \mathrm{L}$ of aqueous phase produced the lowest mean droplet diameter. Hence this volume of oil phase was used for further studies.

\subsection{Optimization of NE Droplet Size by Response Surface} Methodology (RSM). The effect of different volumes of olive oil and polysorbate 80 and different concentrations of polyvinyl alcohol on mean droplet size of NE system was evaluated. Response surface method was used to understand individual as well as combined effect of these parameters. From analysis of variance (ANOVA) (Table 4) it was established that olive oil, polysorbate 80 , and polyvinyl alcohol were highly significant $(P<0.001, P=0.0018$, and $P=0.0359$, resp.) for droplet size of the nanoemulsion system $(P$ values less than 0.05 indicate that model terms are significant). However, combined effect of these factors was 
TABLE 4: ANOVA for response surface quadratic model.

\begin{tabular}{|c|c|c|c|c|c|}
\hline Source & Sum of squares & $\mathrm{df}$ & Mean square & $F$ value & $\begin{array}{c}P \text { value } \\
\text { Prob }>F\end{array}$ \\
\hline Model & 794.6701 & 9 & 88.29667 & 11.81452 & 0.0003 \\
\hline A: olive oil & 528.3974 & 1 & 528.3974 & 70.70214 & $<0.0001$ \\
\hline$B$ : polysorbate 80 & 132.4818 & 1 & 132.4818 & 17.72671 & 0.0018 \\
\hline C: PVA & 43.88657 & 1 & 43.88657 & 5.872235 & 0.0359 \\
\hline$A B$ & 0.452036 & 1 & 0.452036 & 0.060485 & 0.8107 \\
\hline$A C$ & 8.555034 & 1 & 8.555034 & 1.144705 & 0.3098 \\
\hline$B C$ & 0.094241 & 1 & 0.094241 & 0.01261 & 0.9128 \\
\hline$A^{2}$ & 1.474551 & 1 & 1.474551 & 0.197302 & 0.6664 \\
\hline$B^{2}$ & 46.06179 & 1 & 46.06179 & 6.163291 & 0.0324 \\
\hline$C^{2}$ & 36.40223 & 1 & 36.40223 & 4.870796 & 0.0518 \\
\hline Residual & 74.7357 & 10 & 7.47357 & & \\
\hline Lack of fit & 74.7357 & 5 & 14.94714 & & \\
\hline
\end{tabular}

TABLE 5: (a) Temporal stability studies of NESH 01. (b) Temperature stability of NESH 01.

(a)

\begin{tabular}{lcc}
\hline Day & Size $d(\mathrm{~nm})$ & Refractive index \\
\hline 1 & 30 & 1.334 \\
2 & 32 & 1.334 \\
3 & 33 & 1.334 \\
4 & 35 & 1.334 \\
5 & 36 & 1.334 \\
6 & 35 & 1.334 \\
7 & 37 & 1.334 \\
\hline
\end{tabular}

(b)

\begin{tabular}{lc}
\hline Temperature $\left({ }^{\circ} \mathrm{C}\right)$ & Size $d(\mathrm{~nm})$ \\
\hline 20 & 30 \\
30 & 32 \\
40 & 33 \\
50 & 31 \\
60 & 42 \\
70 & 69 \\
80 & 119 \\
90 & 139 \\
100 & 167 \\
\hline
\end{tabular}

not significant $(P=0.81,0.30$, and 0.91 , resp. $)$ for droplet size of the nanoemulsion system. Analysis of variance (ANOVA) for droplet size of the nanoemulsion system shows that model fits well with the significance of $F$-test $=11.81(P=0.0003)$ as given in Table 3 .

The contour plot in Figure 3(a) exhibits the behavior of droplet size $(\mathrm{nm})$ of nanoemulsion with respect to changes in volume of polysorbate 80 and olive oil in the selected range, while PVA concentration being constant $(0.55 \%)$. The optimal values of the selected variables are obtained when moving along the major and minor axis of the ellipse. Higher volume of polysorbate 80 coupled with lower volume of olive oil produced lower droplet size while PVA concentration being constant. The contour plot in Figure 3(b) describes droplet size diameter $(\mathrm{nm})$ with respect to changes in the PVA concentration and olive oil content. The contour plot in Figure 3(c) shows effect on droplet size diameter (nm) of nanoemulsion system with respect to changes in PVA concentration and polysorbate 80 content. The PVA concentration $0.1 \%$ coupled to lower olive oil content $(5 \mu \mathrm{L})$ and higher polysorbate 80 content $(1500 \mu \mathrm{L})$ resulted in the droplet diameter of $50 \mathrm{~nm}$.

\subsection{Physical Stability Studies}

3.5.1. Temporal Stability of NESH 01. The temporal study of the NESH 01 system was studied in terms of droplet diameter and refractive index for a period of seven days. The data shown in Table 5(a) suggests that the NESH 01 is stable for a period of minimum one week with negligible variation in the droplet diameter.

3.5.2. Temperature Stability of NESH 01. The NESH 01 was tested for its temperature stability in the range of $20-100^{\circ} \mathrm{C}$ for a period of one hour. NESH 01 was found to be almost stable up to $60^{\circ} \mathrm{C}$ and the droplet size increases gradually thereafter (Table 5(b)). No similar data on temperature stability are yet available in the literature for comparison.

3.6. Transmission Electron Microscopy (TEM). The droplets in the nanoemulsion appear dark, and the surroundings are bright (Figure 4); a "positive" image was seen using TEM. Some droplet sizes were measured using TEM, as it is capable of point-to-point resolution. The droplet size was in agreement with the results obtained from droplet size analysis using the Zetasizer.

3.7. Antibacterial Activity of NESH 01. The antibacterial activity of NESH 01 was conducted using different cultures procured from MTCC by standard spread plate technique. The results (Table 6) show that free clarithromycin shows 
TABLE 6: Antibacterial activity of NESH 01.

\begin{tabular}{lccc}
\hline MTCC bacterial strains & $\begin{array}{c}\text { Zone of inhibition with free } \\
\text { clarithromycin }\end{array}$ & $\begin{array}{c}\text { Zone of inhibition with NESH 01 } \\
\text { containing clarithromycin }\end{array}$ & $\begin{array}{c}\text { Zone of inhibition with NESH 01 } \\
\text { without clarithromycin }\end{array}$ \\
\hline Bacillus subtilis MTCC 121 & 1.1 & 1.8 & BDL \\
Bacillus megaterium MTCC 428 & 1.4 & 2.3 & BDL \\
Enterobacter aerogenesMTCC 111 & 1.8 & 2.2 & BDL \\
Escherichia coli MTCC 1610 & BDL & BDL & BDL \\
Streptococcus pneumoniae MTCC 655 & 2.0 & 2.4 & BDL \\
Azotobacter chrococcus MTCC 446 & 1.6 & 2.0 & BDL \\
\hline
\end{tabular}

BDL: below detection level.

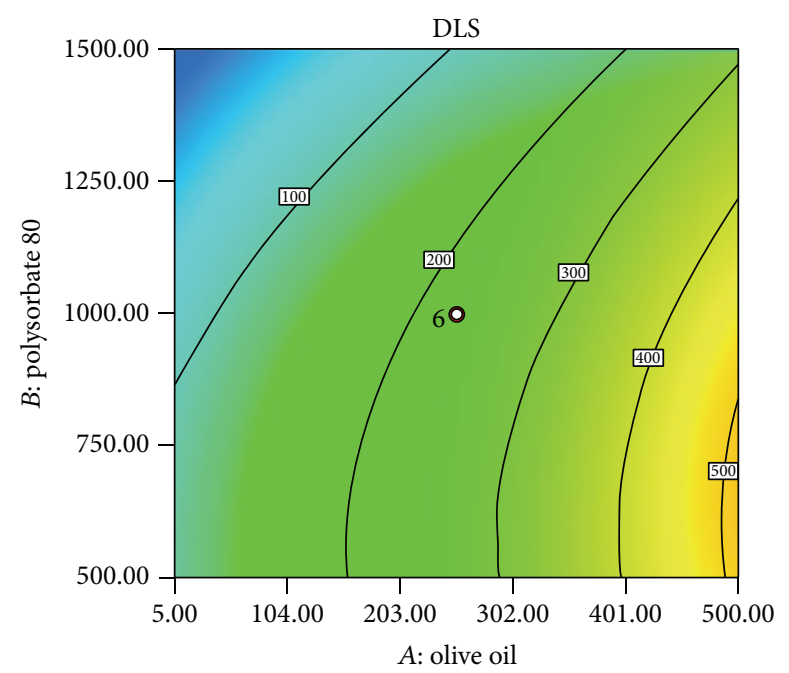

(a)

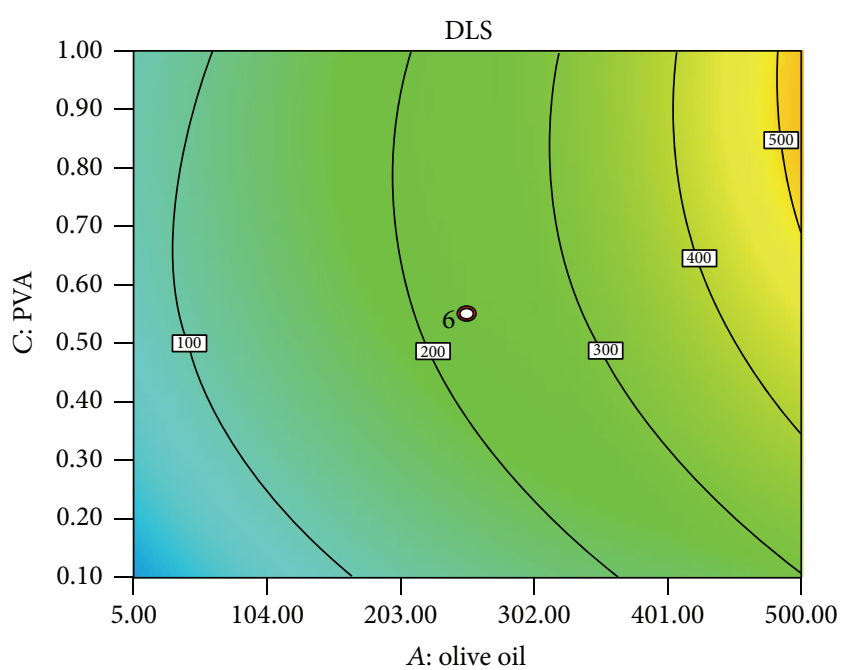

(b)

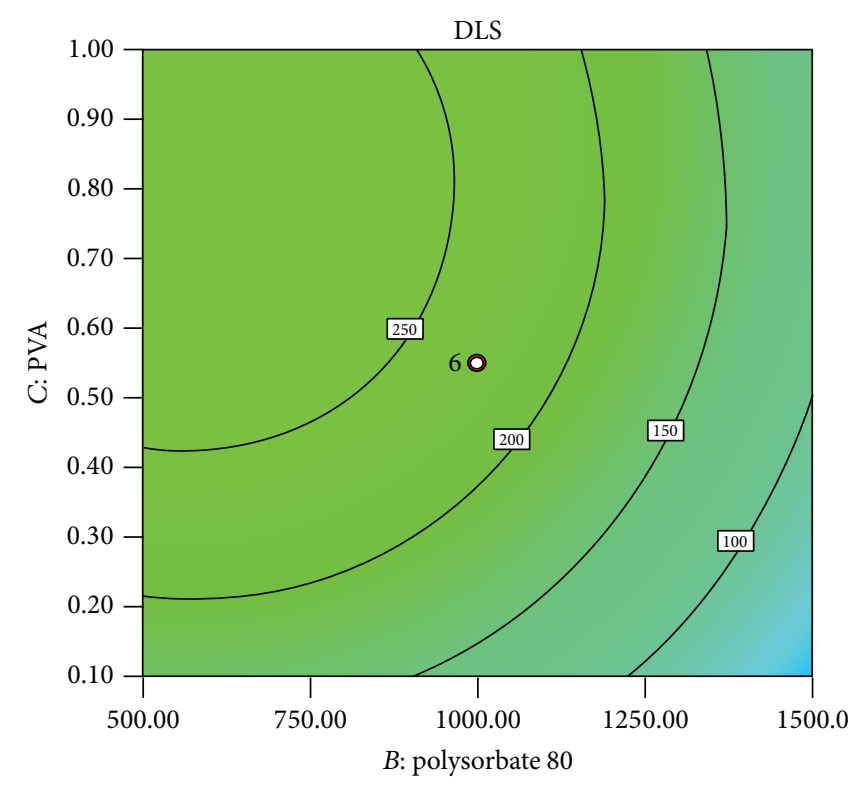

(c)

FIGURE 3: Contour plot showing interaction effect of (a) olive oil and polysorbate 80; (b) olive oil and PVA; and (c) PVA and polysorbate 80 [DLS: dynamic light scattering $(\mathrm{nm})]$. 


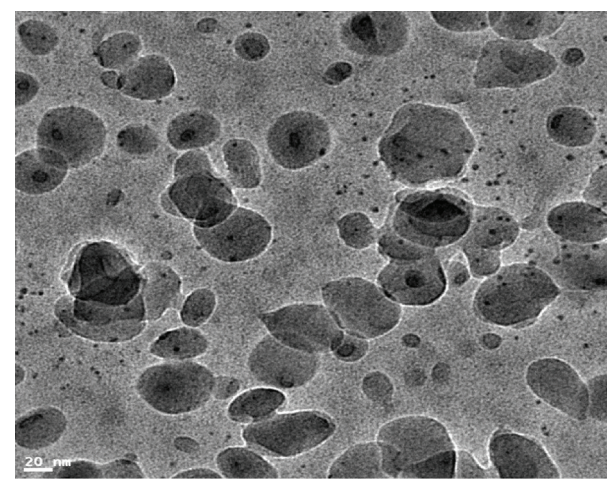

FIGURE 4: TEM photograph of NESH 01.

a smaller zone of inhibition as compared to that of clarithromycin formulated NE (NESH 01). NESH 01 lacking the antibiotic clarithromycin was also tested for the antibacterial activity. However, no zone of inhibition was observed with any of the strains in absence of clarithromycin. This implies that the solubility and hence the bioavailability of the antibiotic clarithromycin increases in a nanoemulsion system like NESH 01.

\section{Conclusion}

Biocompatible oil in water nanoemulsion was fabricated successfully as a vehicle to transport a poorly water soluble drug, clarithromycin. Such nanoemulsions enable the lipophilic drug to be absorbed more rapidly and better when compared with the oil solution. The droplet diameter of the nanoemulsion system was affected dramatically by the type of oil, the oil phase content, type of surfactant, and the temperature. This nanoemulsion system enhanced the solubility $(5 \mathrm{mg} / \mathrm{mL})$ and hence the bioavailability of clarithromycin as evidenced from the increase in zone of inhibition in the antibacterial activity assay. Furthermore, this system was stable for at least one week. In conclusion, the preceding properties of the novel nanoemulsion system NESH 01 postulate its expediency for clinical applications of highly potent sparingly water soluble drugs by enhancing their bioavailability.

\section{Conflict of Interests}

The authors declare that there is no conflict of interests regarding the publication of this paper.

\section{References}

[1] S. Kotta, A. W. Khan, K. Pramod, S. H. Ansari, R. K. Sharma, and J. Ali, "Exploring oral nanoemulsions for bioavailability enhancement of poorly water-soluble drugs," Expert Opinion on Drug Delivery, vol. 9, no. 5, pp. 585-598, 2012.

[2] H. Chen, C. Khemtong, X. Yang, X. Chang, and J. Gao, "Nanonization strategies for poorly water-soluble drugs," Drug Discovery Today, vol. 16, pp. 354-360, 2011.

[3] A. Amani, P. York, H. Chrystyn, and B. J. Clark, "Factors affecting the stability of nanoemulsions-use of artificial neural networks," Pharmaceutical Research, vol. 27, no. 1, pp. 37-45, 2010.

[4] A. M. Mathur, B. Drescher, A. B. Scranton, and J. Klier, "Polymeric emulsifiers based on reversible formation of hydrophobic units," Nature, vol. 392, no. 6674, pp. 367-370, 1998.

[5] S. Tamilvanan and S. Benita, "The potential of lipid emulsion for ocular delivery of lipophilic drugs," European Journal of Pharmaceutics and Biopharmaceutics, vol. 58, no. 2, pp. 357-368, 2004.

[6] H. Yu, C. Shu, L. Chiho et al., "Formulation and evaluation of water-in-oil amoxicillin-loaded nanoemulsions using for Helicobacter pylori eradication," Process Biochemistry, vol. 47, pp. 1469-1478, 2012.

[7] J. Li, S. Nie, X. Yang, C. Wang, S. Cui, and W. Pan, "Optimization of tocol emulsions for the intravenous delivery of clarithromycin," International Journal of Pharmaceutics, vol. 356, no. 1-2, pp. 282-290, 2008.

[8] W. He, Y. Tan, Z. Tian, L. Chen, F. Hu, and W. Wu, "Food protein-stabilized nanoemulsions as potential delivery systems for poorly water-soluble drugs: preparation, in vitro characterization, and pharmacokinetics in rats," International Journal of Nanomedicine, vol. 6, pp. 521-533, 2011.

[9] K. A. Rodvold, "Clinical pharmacokinetics of clarithromycin," Clinical Pharmacokinetics, vol. 37, no. 5, pp. 385-398, 1999.

[10] P. P. Constantinides, A. Tustian, and D. R. Kessler, "Tocol emulsions for drug solubilization and parenteral delivery," Advanced Drug Delivery Reviews, vol. 56, no. 9, pp. 1243-1255, 2004.

[11] A. X. Chen, "Macrolides containing oil-in-water emulsions," W/O Patent 2005/016308 A1, 2005.

[12] K. Chauhan, U. Trivedi, and K. C. Patel, "Application of response surface methodology for optimization of lactic acid production using date juice," Journal of Microbiology and Biotechnology, vol. 16, no. 9, pp. 1410-1415, 2006.

[13] H. Pathak, D. Kantharia, A. Malpani, and D. Madamwar, "Naphthalene degradation by Pseudomonas sp. HOB1: in vitro studies and assessment of naphthalene degradation efficiency in simulated microcosms," Journal of Hazardous Materials, vol. 166, no. 2-3, pp. 1466-1473, 2009.

[14] Z. Syafinaz, B. Mahiran, B. Hamidon et al., "Formulation optimization of a palm-based nanoemulsion system containing levodopa," International Journal of Molecular Sciences, vol. 13, pp. 13049-13064, 2012.

[15] M. Trotta, F. Pattarino, and T. Ignoni, "Stability of drug-carrier emulsions containing phosphatidylcholine mixtures," European Journal of Pharmaceutics and Biopharmaceutics, vol. 53, no. 2, pp. 203-208, 2002. 

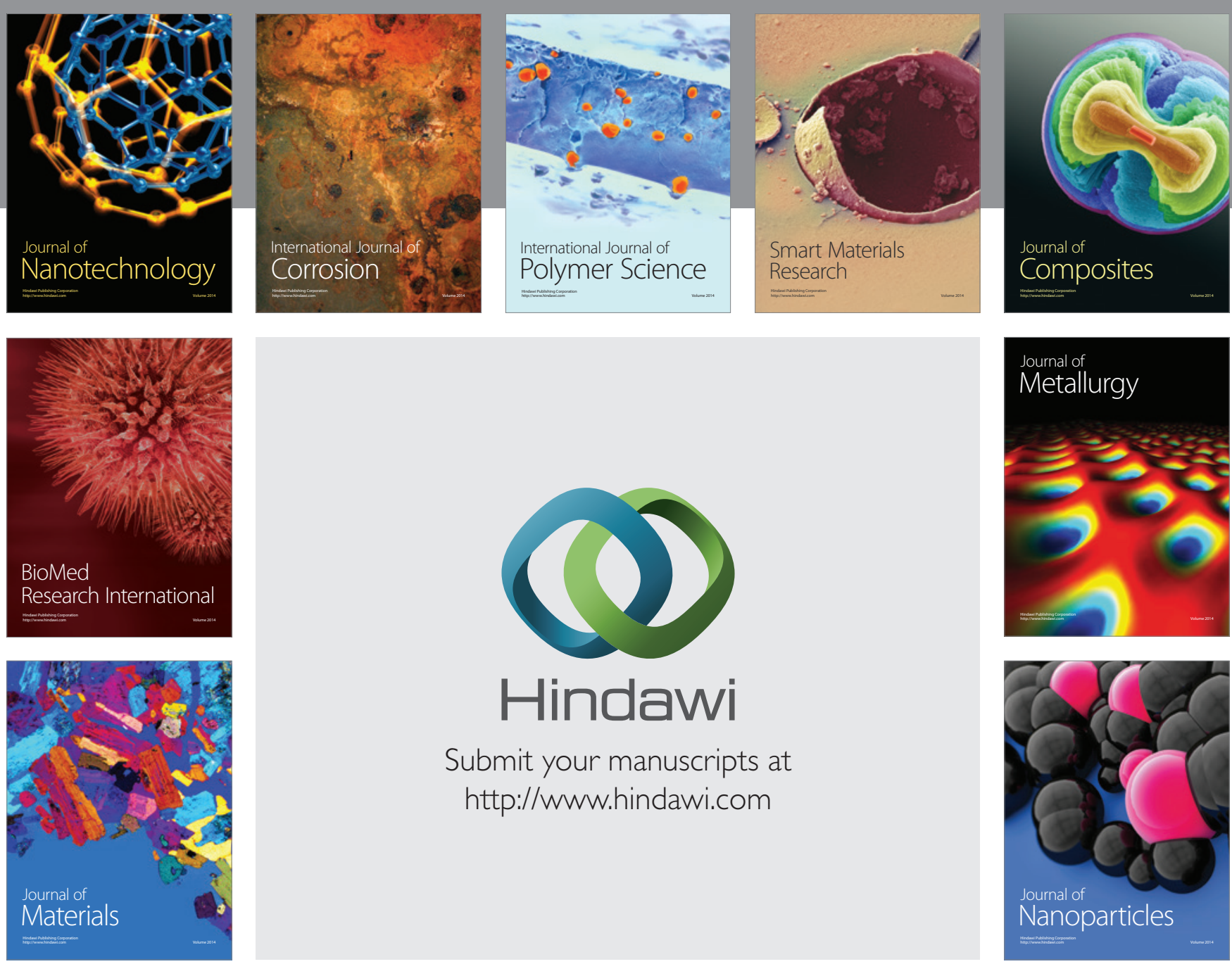

Submit your manuscripts at http://www.hindawi.com
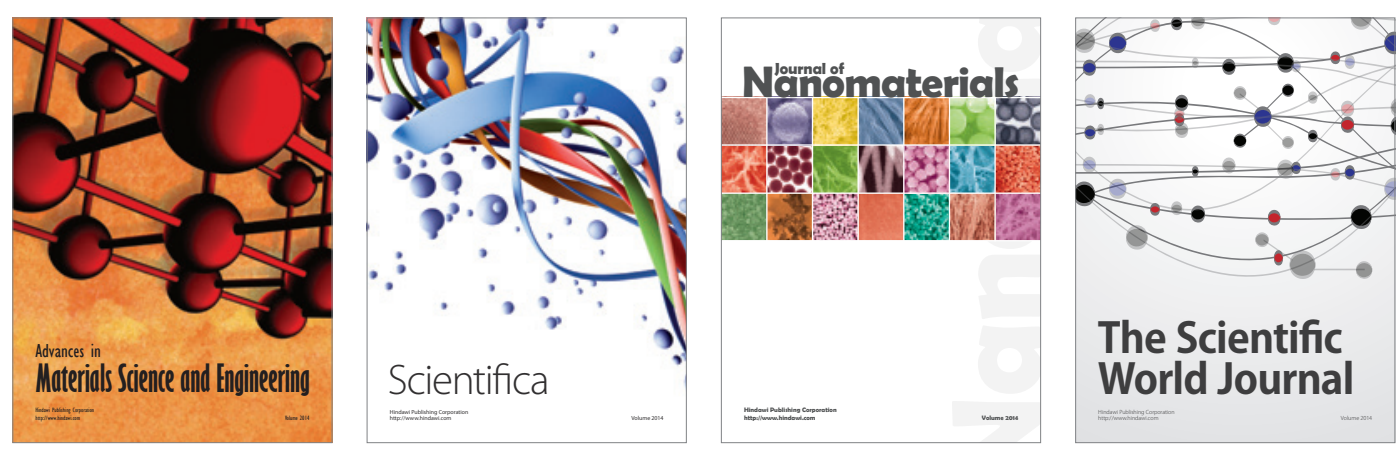

\section{The Scientific World Journal}
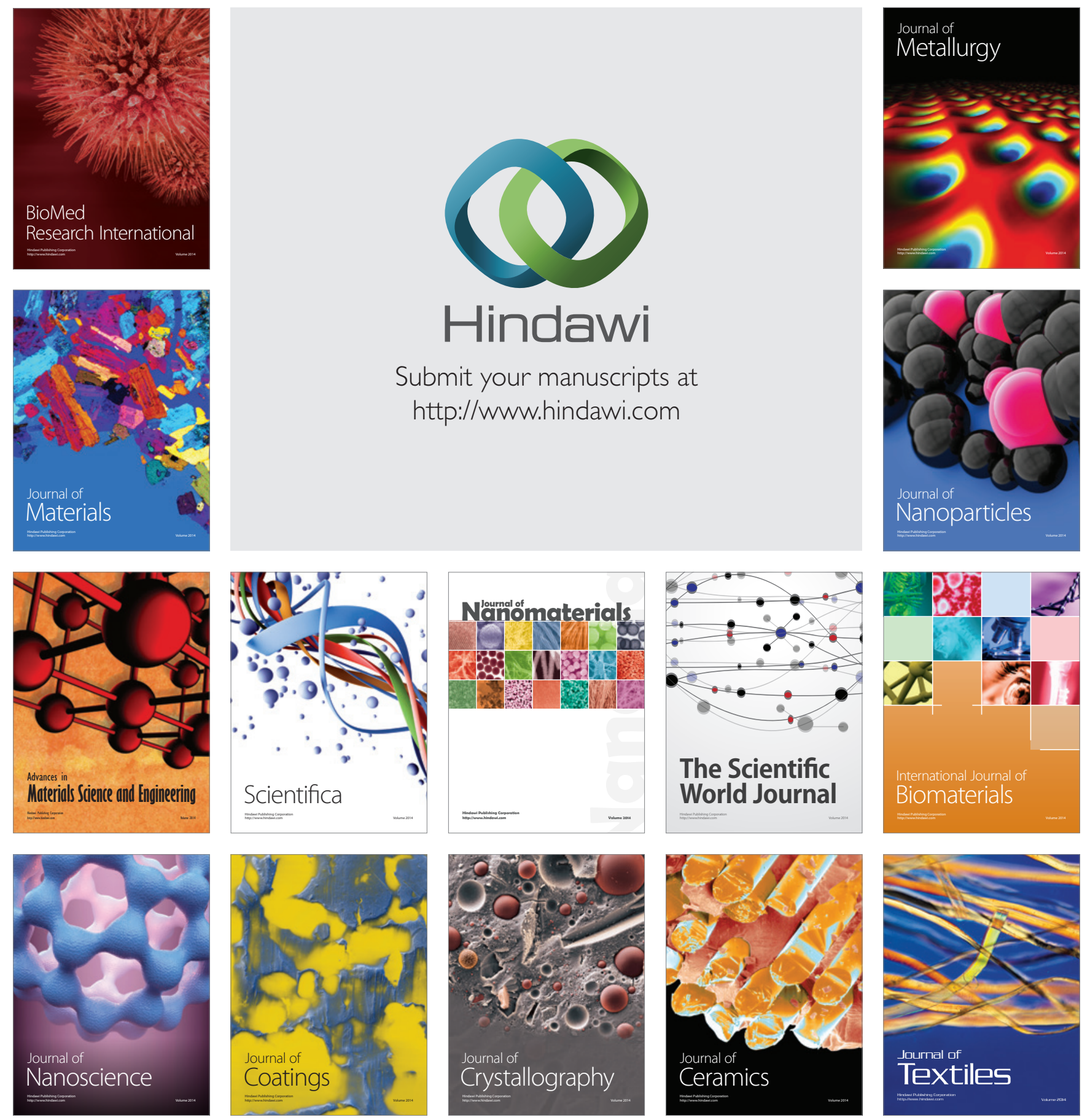